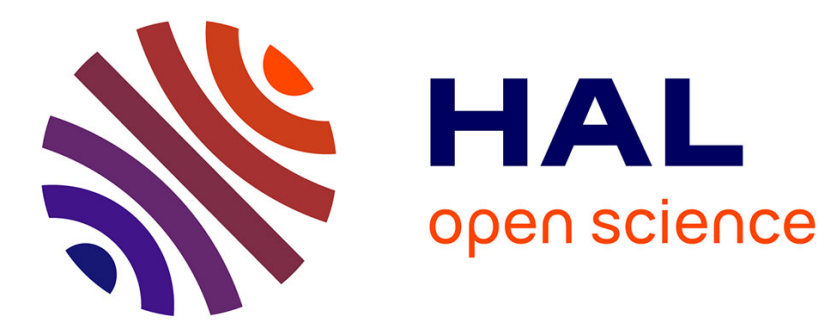

\title{
Mechanical and acoustic properties of macroporous acrylate materials near glass transition
}

\author{
Artem Kovalenko, Thomas Brunet, Olivier Mondain-Monval
}

\section{To cite this version:}

Artem Kovalenko, Thomas Brunet, Olivier Mondain-Monval. Mechanical and acoustic properties of macroporous acrylate materials near glass transition. Polymer, 2018, 148, pp.239 - 246. 10.1016/j.polymer.2018.06.033 . hal-01894105

\section{HAL Id: hal-01894105 \\ https://hal.science/hal-01894105}

Submitted on 12 Oct 2018

HAL is a multi-disciplinary open access archive for the deposit and dissemination of scientific research documents, whether they are published or not. The documents may come from teaching and research institutions in France or abroad, or from public or private research centers.
L'archive ouverte pluridisciplinaire HAL, est destinée au dépôt et à la diffusion de documents scientifiques de niveau recherche, publiés ou non, émanant des établissements d'enseignement et de recherche français ou étrangers, des laboratoires publics ou privés. 


\section{TOC:}

Master curve

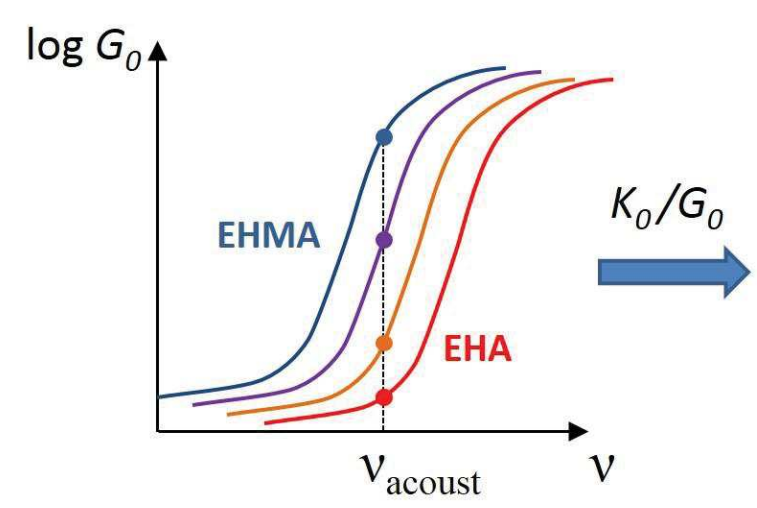

Macroporous polymer

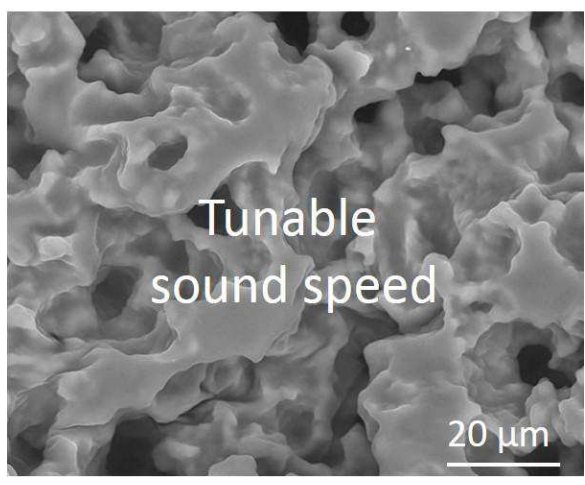




\section{TOC:}

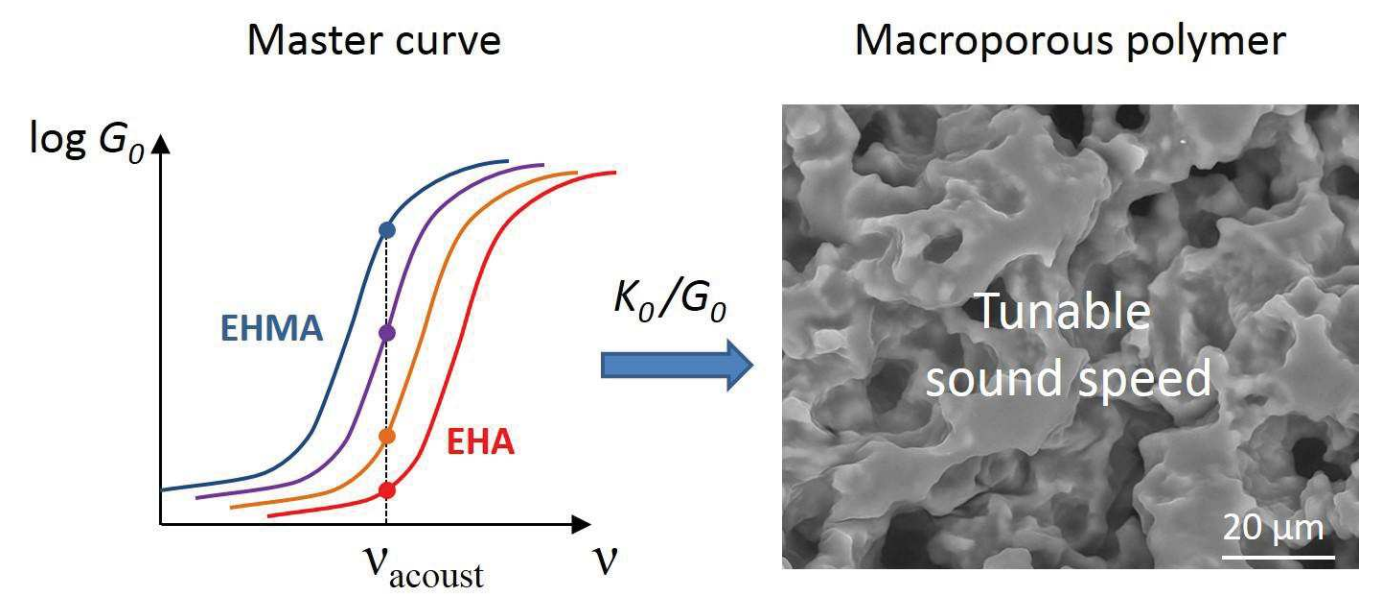




\title{
Mechanical and acoustic properties of macroporous acrylate materials near glass transition
}

Artem Kovalenko ${ }^{1,2^{*}}$, Thomas Brunet ${ }^{3}$, Olivier Mondain-Monval ${ }^{1 *}$

${ }^{1}$ - University of Bordeaux - CNRS, Centre de Recherche Paul Pascal, Pessac, France

2 - Present address: École Supérieure de Physique et de Chimie Industrielles de la Ville de Paris (ESPCI) ParisTech, CNRS UMR 7615, Paris, France

3 - University of Bordeaux - CNRS - Bordeaux INP, Institut de Mécanique et d'Ingénierie, Talence, France

* - corresponding authors

\begin{abstract}
:
We study the evolution of the acoustic properties of porous polymer materials in the glass transition temperature domain. First, we study the cross-linked 2-ethylhexyl acrylate (EHA) 2-ethylhexyl methacrylate (EHMA) non-porous matrices at various compositions and, consequently, various glass transition temperatures. Based on the frequency-temperature equivalence principle, we build master curves in order to determine the bulk $\left(K_{0}\right)$ and shear $\left(G_{0}\right)$ moduli corresponding to ultrasonic frequencies (about $300 \mathrm{kHz}$ ). Next, we synthesize some porous samples of these matrices using the emulsion-templated polymerization approach usually known as polyHIPE (for polymerized High Internal Phase Emulsion). We measure the longitudinal sound speed $C_{L}$ for different matrix compositions (at room temperature) or at different temperatures (for the same sample composition). As expected, we show that the sound speed decreases with increasing $K_{0} / G_{0}$ ratio, i.e. as matrices become softer, which is either due to an increase of EHA content or to an increase of the temperature. This demonstrates how composition and temperature may be used to tune the sound speed in porous polymers. We show that the theoretical predictions using a low-frequency multiple scattering model leads to underestimated values of the sound speed, especially for soft matrix. We attribute this discrepancy to the difference in mechanical properties of the polymer matrix between non porous and porous samples.
\end{abstract}

\section{Keywords:}

Porous polymers, polyHIPEs, sound speed, glass transition, time-temperature equivalence. 


\section{Introduction}

Polymer foams are used in a wide range of applications ranging from packaging to solidsupported catalysis ${ }^{1}$, shock absorbers ${ }^{2}$, thermal insulator devices ${ }^{3}$, filters and membranes ${ }^{4}$. Such foams are also very attractive for acoustics ${ }^{5}$ due to the possibility to tune the sound reflection, attenuation and transmission by varying polymer chemical nature or processing methods. For example, polymer foams may either be shaped in anechoic devices (as in the famous "Alberich" materials ${ }^{6}$ set up by the Germans during World War 2) or microscopically structured, as in more recent works on locally resonant acoustic metamaterials ${ }^{7}$. Sound propagation in polymer foams is a direct function of the matrix porosity and mechanical properties. A polymer matrix should be considered as soft (respectively hard) when its glass transition temperature $\left(T_{\mathrm{G}}\right)$ is much lower (respectively higher) than the temperature at which the measurements are performed. For a soft porous material in the low-frequency limit (the sound wavelength is much larger than the pore size), the following expression can be used to describe the evolution of the longitudinal sound velocity $C_{\mathrm{L}}$ as a function of the foam porosity $\phi$ and elastic properties ${ }^{2,8}$ :

$$
C_{\mathrm{L}}=\frac{C_{0}}{\sqrt{\left(1+\frac{3 K_{0}}{4 G_{0}} \Phi\right)}}
$$

where $C_{0}, K_{0}$ and $G_{0}$ are, respectively, the sound velocity, the bulk and shear moduli of the non-porous polymer matrix. Equation (1) may be used for low and intermediate porosities ( $\phi$ $<50 \%$ ) only and qualitatively accounts for the observed spectacular difference ${ }^{9}$ between the sound speeds in soft (polydimethylsiloxane (PDMS) matrix) and hard (polystyrene (PS)) foams. In the case of PDMS, the matrix has high $K_{0} / G_{0}$ ratio $\left(\sim 10^{3}\right)$ which leads to very low values of longitudinal sound velocity $C_{L}$, well below $100 \mathrm{~m} / \mathrm{s}$ even at rather low porosities (about 30\%). By contrast, polystyrene matrix with low values of the $K_{0} / G_{0}$ ratio $(\sim 1)$ leads to values typically ten times larger. Thus, glass transition in polymer foams should be followed by a drastic change in sound speed from low (rubbery state) to high values (glassy state). However, compared to non-porous polymers ${ }^{10,11,12,13}$, the studies relating mechanical properties to the sound speed in porous polymers near $T_{\mathrm{G}}$ are limited ${ }^{14,15}$.

The aim of this paper is to study the evolution of the acoustic properties of macroporous polymer materials in the vicinity of the glass transition temperature domain. The mechanical moduli of the matrix depend on the composition, temperature and frequency of the measurement. We rely on the temperature-frequency equivalence principle widely used in literature to describe the mechanical properties of polymers. To explore a gradual change in properties, we vary either the polymer composition or the measuring temperature while keeping the other parameters constant. To tune the $T_{\mathrm{G}}$ of our samples, we choose a crosslinked network composed of statistically copolymerized monomers with different $T_{G}$ values $^{16},{ }^{17}$. As the soft component, we use 2-ethylhexyl acrylate (EHA, $T_{\mathrm{G}} \sim-58^{\circ} \mathrm{C}$ ) which gives a polymer with low shear modulus $\left(G_{0} \leq 1 \mathrm{MPa}\right)$ not only at low ("mechanical") frequencies, but also at high frequencies corresponding to ultrasonic measurements (about $300 \mathrm{kHz}$ ). The second component is 2-ethylhexyl methacrylate (EHMA, $T_{\mathrm{G}} \sim-10^{\circ} \mathrm{C}$ ) which 
corresponds to a mechanically soft but acoustically much more elastic polymer ( $G_{0}$ about 120 $\mathrm{MPa}$ at $300 \mathrm{kHz}$ frequency). The networks with different monomer ratios are synthesized by radical photopolymerization with a similar amount (about $4 \%$ wt.) of cross-linker ethylene glycol dimethacrylate (EGDMA). In a second set of experiments, we study the evolution of the acoustic properties as a function of temperature in a sample with constant given porosity and polymer matrix composition.

First, we obtain non-porous samples with various $T_{\mathrm{G}}$ values and characterize their properties by dynamic mechanical analysis (DMA), differential scanning calorimetry (DSC) and sound velocity measurements. Next, we synthesize porous samples with controlled porosity and porous structure using the emulsion-templated polymerization approach usually known as polyHIPE (for polymerized High Internal Phase Emulsion). We characterize the structure and porosity of the samples, which are similar for all matrix compositions. Finally, we explore the dependence of sound velocity on the matrix composition and on temperature. As expected, we show that sound velocity decreases with increasing $K_{0} / G_{0}$ ratio, i.e. as matrices become softer. However, direct use of a simple multiple scattering model (equation (1)) leads to underestimated values of the sound speed, especially for soft matrix. We discuss this discrepancy that we attribute to the different mechanical properties of the polymer matrices obtained with or without the presence of water and surfactant molecules in the system.

\section{Experimental}

\subsection{Materials}

All reagents including 2-ethylhexyl methacrylate (EHMA), 2-ethylhexyl acrylate (EHA) and ethyleneglycol dimethyl methacrylate (EGDMA) monomers, sorbitan laurate surfactant (Span 20), radical photoinitiator 2,2-dimethyl-2-hydroxyacetophenone (Darocure 1173) and $\mathrm{NaCl}$ were obtained from Sigma Aldrich and used without purification.

\subsection{Preparation of the samples of various EHMA/EHA ratios}

Table 1 presents the compositions of the monomer mixtures used for the synthesis of both non-porous and porous materials. We chose four different compositions with different ratios of comonomers (EHMA and EHA), while the concentration of cross-linker (EGDMA) and radical photoinitiator (Darocure 1173) were maintained constant and equal to $4 \%$ for both. These mixtures were used without modification for the synthesis of non-porous samples. To obtain porous samples, water-in-oil emulsions were stabilized by adding $4 \mathrm{~g}$ of surfactant (Span 20) to $10.4 \mathrm{~g}$ of the monomer mixture and further incorporation of $10 \mathrm{~g}$ of the water phase (1.5\% wt. $\mathrm{NaCl}$ solution in MiliQ water). This amount of water phase corresponds to about $40 \%$ wt. of the total mass. The mixture was homogenized using a UltraTurrax turbine for $10 \mathrm{~min}$ at $3000 \mathrm{rpm}$. The size distributions of emulsions were obtained by static light scattering performed in a Mastersizer S apparatus (Malvern) with dodecane as the continuous phase. In dodecane, emulsion droplets remain fully dispersed. 
Table 1. Compositions (by weight) of monomer mixtures used for the synthesis. Glass transition temperatures $T_{\mathrm{G}}$ of the corresponding matrices determined using differential scanning calorimetry (DSC).

\begin{tabular}{c|c|c|c|c}
\multirow{2}{*}{} & \multicolumn{4}{|c}{ Samples } \\
\cline { 2 - 5 } & $\mathrm{X} 0$ & $\mathrm{X} 04$ & $\mathrm{X} 07$ & $\mathrm{X} 1$ \\
\hline EHMA / EHA ratio & $0 / 100$ & $40 / 60$ & $70 / 30$ & $100 / 0$ \\
\hline EHA $(\mathrm{g})$ & 9.6 & 5.76 & 2.88 & 0 \\
EHMA $(\mathrm{g})$ & 0 & 3.84 & 6.72 & 9.6 \\
EGDMA $(\mathrm{g})$ & 0.4 & 0.4 & 0.4 & 0.4 \\
\hline Total $(\mathrm{g})$ & 10 & 10 & 10 & 10 \\
\hline$T_{\mathrm{G}}$ from DSC $\left({ }^{\circ} \mathrm{C}\right)$ & -65 & -55 & -35 & -10
\end{tabular}

\subsection{Polymerization}

Monomer mixtures (for non-porous samples) or water-in-oil emulsions (for porous samples) were put in flat Teflon molds having $50 \mathrm{~mm} \times 50 \mathrm{~mm} \times 2 \mathrm{~mm}$ wells and covered with a thin Teflon lid. The Teflon was necessary for preventing strong adhesion to the mold which may cause macroscopic holes inside samples due to the polymerization-induced contraction. The thickness of the Teflon was small enough to allow for the photopolymerization, which was triggered by Dymax BlueWave 200 UV lamp. After 15 min of polymerization, the samples were unmolded and, in the case of porous samples, dried at room temperature until a constant weight was achieved.

\subsection{Characterization}

The density of the matrix was measured using an AccuPyc 1330 helium pycnometer (Micromeritics). The porosity was measured by directly measuring the mass and volume of the samples.

The glass transition temperatures were obtained from differential scanning calorimetry (DSC) experiments performed on non-porous samples. In DSC, the samples were rapidly cooled down to $-140^{\circ} \mathrm{C}$ and heated at the speed of $20^{\circ} \mathrm{C} / \mathrm{min}$. The obtained curves (Figure 1) show that both the $T_{\mathrm{G}}$ and the temperature interval of glass transition increase with EHMA content. The transition is particularly broad for the pure EHMA sample X1 (please note that, for such broad transitions, the obtained values cannot be very accurately determined). Table 1 reports the $T_{\mathrm{G}}$ values taken as half-vitrification temperatures on the DSC curves.

The porous structures of the obtained samples were characterized with a TM-3030 scanning electron microscope (Hitachi). The observations were done on freshly fractured samples surfaces covered by a thin sputtered gold layer. 


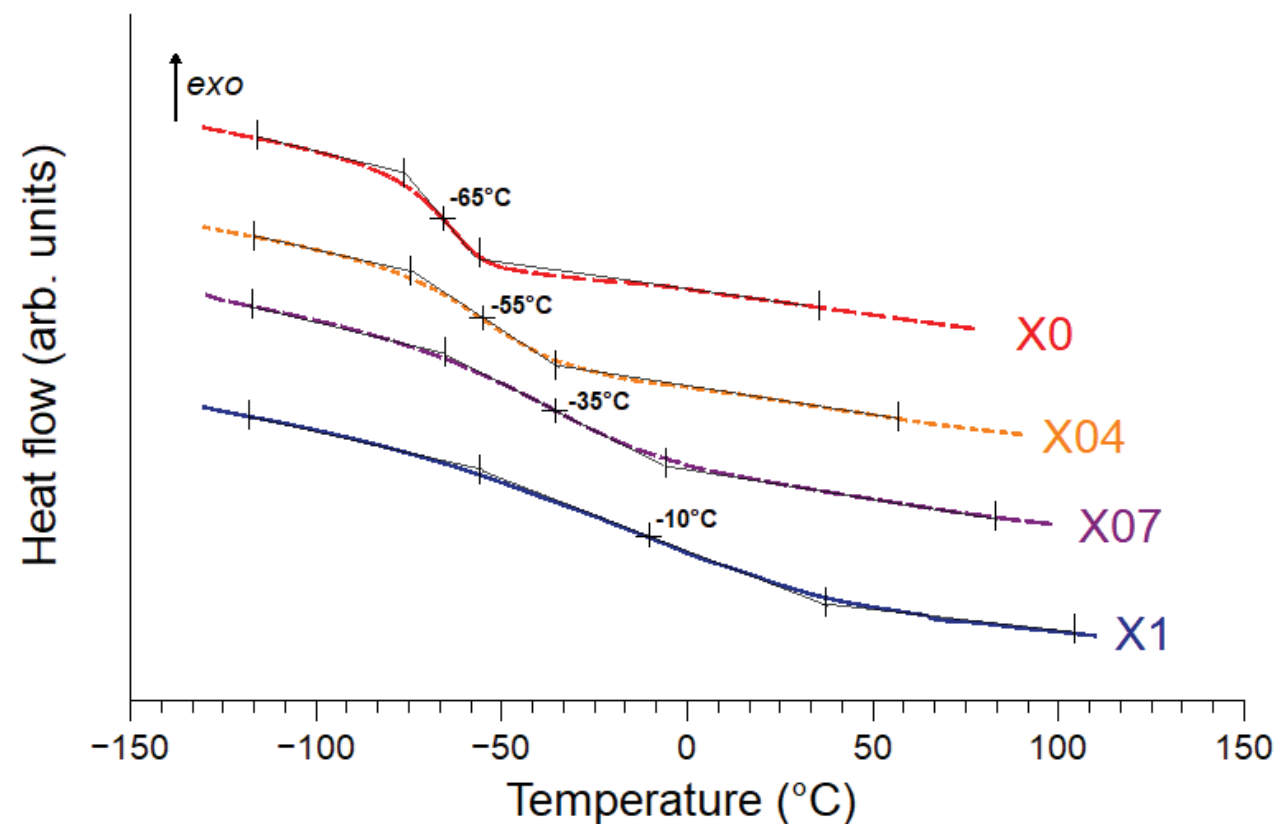

Figure 1 - Differential scanning calorimetry curves showing the glass transition region for four non-porous polymer matrices with various EHMA/EHA ratios (see Table 1).

\subsection{Mechanical properties}

The shear moduli of non-porous samples were measured using a Dynamic Mechanical Analysis apparatus (DMA/SDTA861e, Mettler Toledo). Two equivalent samples with dimensions $5 \mathrm{~mm} \times 5 \mathrm{~mm} \times 2 \mathrm{~mm}$ were cut from the original samples and submitted to shear oscillations with frequency varying from 1 to $100 \mathrm{~Hz}$ and shear strain amplitudes $\gamma_{\max } \sim$ 0.0025 , which is in the linear domain. The shear stress $\sigma_{\tau}$ was registered as function of applied shear strain $\gamma$. This allows determining the real and imaginary parts of the shear modulus $G$ :

$$
\sigma_{\tau}=\left(G^{\prime}+i G^{\prime \prime}\right) \gamma
$$

To obtain the master curves, the measurements were conducted at ten different temperatures between $T_{\mathrm{G}}-40^{\circ} \mathrm{C}$ and $T_{\mathrm{G}}+50^{\circ} \mathrm{C}$. The samples were first cooled down in the DMA apparatus to a temperature below the glass transition, tightened in the clamps and then tested at a constant temperature. The equilibration time at each temperature was at least $20 \mathrm{~min}$. The obtained curves were used for mastering as described in the result section. The reproducibility was checked by repeating the measurements on 3 equivalent samples.

\subsection{Acoustic properties}

The sound speeds were obtained from direct contact measurements on two samples with different thicknesses ( 6 and $12 \mathrm{~mm}$ for non-porous samples, 2 and $4 \mathrm{~mm}$ for porous samples), as described in our previous papers $8,9,21$. Each sample was placed between two identical broadband ultrasonic (US) transducers (emitter and receiver, Olympus V301) with a diameter of $30 \mathrm{~mm}$ and a central frequency of $500 \mathrm{kHz}$. The emitting transducer was excited with short (broadband) pulses generated by a pulser/receiver (Olympus, 5077PR-40-E) that was also 
used to amplify the electric signal recorded by the receiving transducer before its acquisition on a computer via an oscilloscope (Lecroy, 9450A). Then, Fast Fourier Transforms (FFTs) were performed on these two gated signals to extract the corresponding phase and amplitude spectra. Knowing the thickness difference between two samples, the phase velocity was obtained. The obtained values were found to be only slightly dependent on the sound frequency within the full width-half maximum bandwidth. Thus, the value at $300 \mathrm{kHz}$ was used for further calculations.

Using the values of the sound speed for non-porous samples $C_{0}$, and of their density $\rho$ and shear modulus $G_{0}$ ' at $f=300 \mathrm{kHz}$, we calculated the corresponding bulk modulus $K_{0}$ using the following equation:

$$
K_{0}=\rho C_{0}^{2}-4 / 3 G_{0}
$$

We also measured the temperature dependence of the acoustical signal using a simple setup shown in Figure 2. The transducers and the sample were covered by a silicone heating element connected to a controller. Before each acoustical data acquisition, the setup was left to stabilize for at least $30 \mathrm{~min}$ and until no further change in acoustic signal was obtained. Temperature measurements using a flexible thermocouple have shown that the stationary temperature distribution in the part of the setup located under the heating element is homogeneous and stable in time.
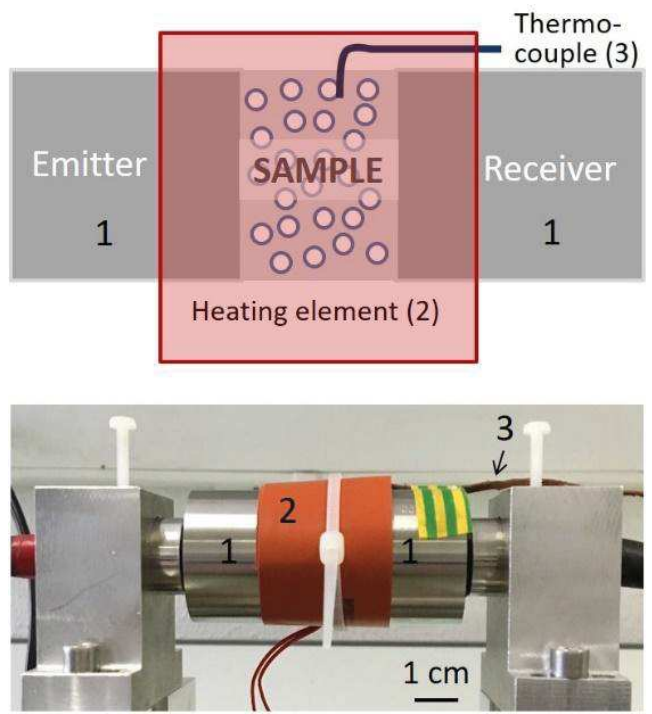

Figure 2 - Experimental setup for acoustical measurements at different temperatures.

\section{Results and discussion}

The current section is organized in the following manner. First, we measure the mechanical and acoustic properties of non-porous EHMA-EHA samples with four different monomer ratios. Second, we describe the structure of porous materials obtained with these four matrices. In the final part, we compare the acoustic properties of the porous samples as a 
function of matrix composition (at constant temperature) and, finally, as a function of temperature (at constant composition).

\subsection{Properties of the non-porous polymer matrices}

Table 2 summarizes the properties of the polymer matrices measured on the non-porous samples.

All samples have similar densities, about $1000 \mathrm{~kg} / \mathrm{m}^{3}$, as measured by helium pycnometry.

Table 2 - Properties of non-porous samples at $20^{\circ} \mathrm{C}$ (the values in italic are calculated as described in the text).

\begin{tabular}{c|c|c|c|c} 
Samples & $\mathrm{X} 0$ & $\mathrm{X} 04$ & $\mathrm{X} 07$ & $\mathrm{X} 1$ \\
\hline$\rho, \mathrm{kg} / \mathrm{m}^{3}$ & $994 \pm 1$ & $995 \pm 1$ & $1000 \pm 1$ & $1006 \pm 1$ \\
$G_{0}^{\prime}(1 \mathrm{~Hz}), \mathrm{MPa}$ & $0.075 \pm 0.01$ & $0.2 \pm 0.05$ & $0.4 \pm 0.1$ & $2 \pm 0.5$ \\
$G_{0}{ }^{\prime}(300 \mathrm{kHz}), \mathrm{MPa}$ & 1 & 6.3 & 38 & 120 \\
$C_{0}, \mathrm{~m} / \mathrm{s}$ & $1520 \pm 20$ & $1530 \pm 20$ & $1560 \pm 20$ & $1850 \pm 20$ \\
$K_{0}(300 \mathrm{kHz}), \mathrm{MPa}$ & 2300 & 2320 & 2380 & 3280
\end{tabular}

Mechanical properties of the EHMA-EHA materials depend strongly on temperature and measurement frequency $f$. We used the temperature-frequency equivalence principle ${ }^{18}$ to build the master curves from the mechanical measurements of the real part of the shear modulus $G_{0}$ ' at different temperatures via a commonly accepted procedure. Briefly, the values of $G_{0}$ ' were measured at frequencies in the $1-100 \mathrm{~Hz}$ range and were plotted in the double logarithmic scale $\log \left(G_{0}{ }^{\prime}\right)-\log (f)$. The segment at $20^{\circ} \mathrm{C}$ was chosen as a reference. For other temperature segments, a shift factor $a(T)$ was applied for the frequencies in order to obtain a continuous line for $G_{0}$ '. The coherence of mastering was checked using imaginary part $G_{0}$ "' (See Supporting Information, Figure SI-1). The obtained $G_{0}{ }^{\prime}$ master curves for the four polymer matrices X0, X04, X07 and X1 are compared in Figure 3.

At low frequencies (or high temperatures), the modulus tends to low values (below $1 \mathrm{MPa}$ ) that are typical for the rubbery state of polymers. At high frequencies, the modulus attains high values (hundreds of MPa), typical for glassy state. Due to the higher glass transition temperature of EHMA, the increase of the EHMA / EHA ratio shifts the glass transition to lower frequencies. Table 2 presents the values of $G_{0}$ ' at acoustic frequency $f=300 \mathrm{kHz}$. These values range from $0.8 \mathrm{MPa}$ to $150 \mathrm{MPa}$, for $\mathrm{X} 0$ and $\mathrm{X} 1$, respectively. Despite very similar rubber-like properties at low ("mechanical") frequencies, the master curves predict very different behavior at acoustic frequencies with a difference of almost two orders of magnitude in the shear moduli between $\mathrm{X} 0$ and $\mathrm{X} 1$.

The measurements of the longitudinal sound velocity have shown the increase of $C_{0}$ with the EHMA fraction passing from $1520 \mathrm{~m} / \mathrm{s}$ for $\mathrm{X} 0$ to $1850 \mathrm{~m} / \mathrm{s}$ for X1. This is consistent with the higher shear modulus of EHMA with respect to EHA. 


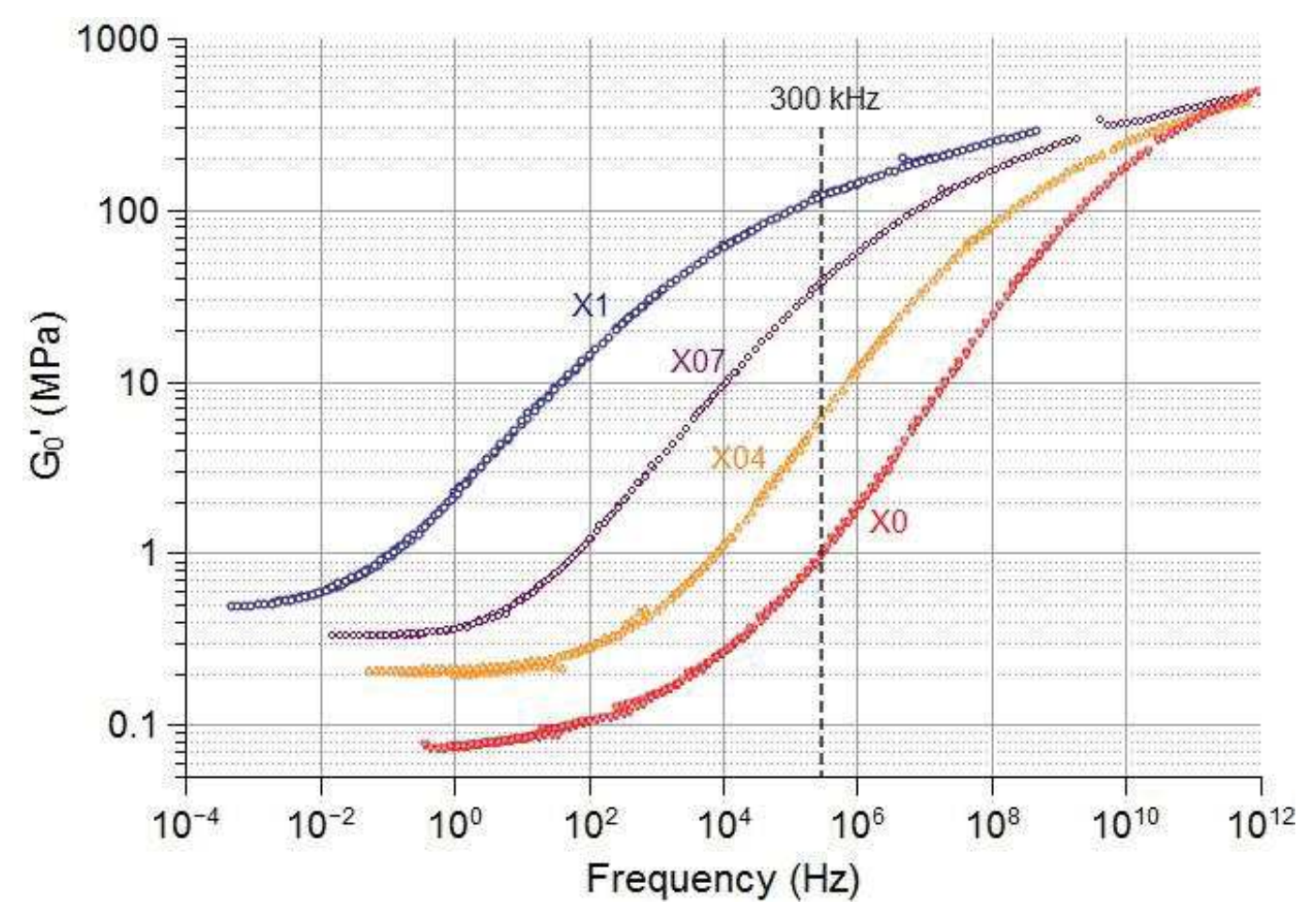

Figure 3 -Master curves of the real part of the shear modulus $G_{0}$ ' for four polymer matrices with various EHA-EHMA ratios (see Table 1) obtained from the frequency-temperature equivalence principle with reference temperature equal to $20^{\circ} \mathrm{C}$. The dashed vertical line corresponds to the acoustic frequency.

The values of bulk modulus $K_{0}$ calculated using equation 4 increase with the EHMA content and vary from 2300 to $3280 \mathrm{MPa}$, which corresponds to an intermediate behavior between the soft PDMS rubber and glassy polystyrene studied previously ${ }^{9}$.

\subsection{Emulsion templating and corresponding porous structure}

For all EHMA/EHA ratios, the size distribution and morphology of water-in-oil emulsions as well as the porous structure of the samples were similar to the case of pure EHA, represented in Figure 4. The optical microscopy pictures of the water-in-oil emulsion (Figure 4a) reveals its strongly aggregated morphology. The measurements of size distributions of droplets dispersed in dodecane (Figure $4 \mathrm{~b}$ ) evidence a monomodal distribution with a maximum value increasing from 3 to $7 \mu \mathrm{m}$ when increasing the EHMA/EHA ratio. Since no distinct droplets of this size are observed in the water-in-monomer emulsions, we suggest a chain-like aggregated structure of the droplets when dispersed in the monomer solutions, as schematized in the inset of Figure 4a. The "elemental" droplets inside the aggregates have a diameter on the order of $5 \mu \mathrm{m}$ and their shape is probably distorted by a layer of poorly soluble Span 20 surfactant.

Similar chain-like aggregated morphology is found in the pore structure shown on scanning electron microscopy (SEM) images in Figure $4 c$ and $d$. This structure of the final material might be compared to the one obtained in silicone polyHIPEs studied in our group ${ }^{9}$. In both cases, the aggregated structure of the initial emulsion leads to open pore materials without a very high internal phase fraction of the polyHIPE (less than 50\%). This is important to avoid 
the collapse of the samples with soft matrix during drying. However, the difference in pore shape between silicone and EHMA-EHA materials is very striking. While silicone polyHIPEs possess pores of spherical shape ${ }^{9}$, the ones observed in Figure $4 \mathrm{~d}$ appear totally non-spherical and with a fully random shape. Such a difference is most probably a consequence of the chemical nature of the used monomers. Silicone monomers do present a much higher hydrophobicity than the acrylate ones. As a consequence, the polymerization of acrylate should be much more perturbed by the presence of water than silicone monomers. As discussed further, we believe that it also impacts the mechanical properties of the polymer walls.
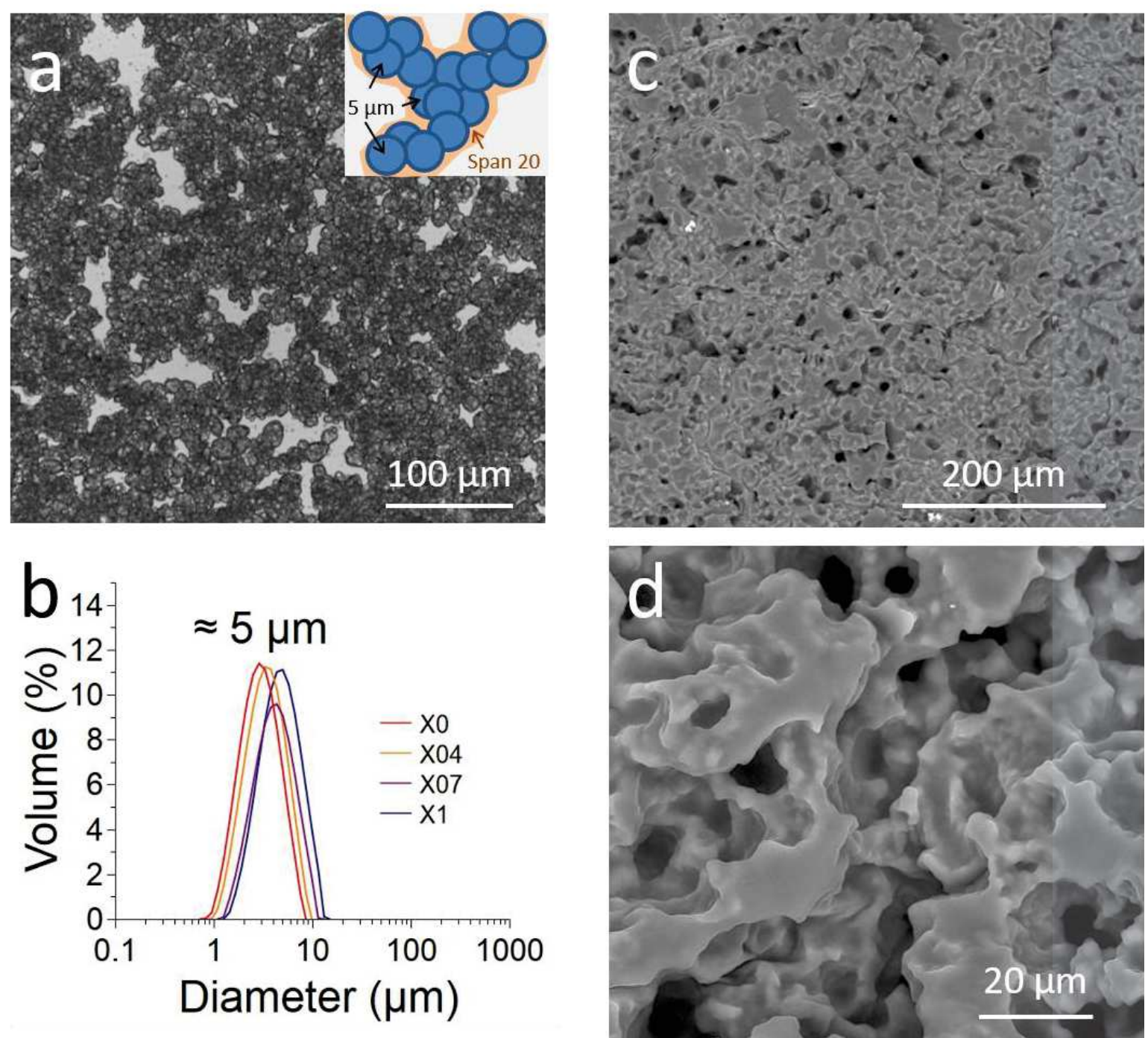

Figure 4 - a) Optical microscopy image of typical $40 \%$ wt. water-in-oil emulsion (composition XO in the present case) used for the synthesis (the inset shows the suggested aggregated emulsion structure); b) emulsion droplet size distribution obtained by static light scattering in dodecane; c) and d) scanning electron microscopy images showing the porous structure of a typical emulsion-templated sample (composition X0) after drying.

From the density measurements, we calculated the porosity of the samples, which increases from 22 to $30 \%$ while increasing the EHMA content from 0 to 100\% (see Table 3). These 
porosities are lower than the water volume fraction introduced in the initial emulsions $(\approx 40$ $\%$ ), which indicates a partial collapse of the pores. This might be caused by two effects. First, some part of the structure may be isolated from the percolated pore network. These pores inside the soft matrix will shrink and collapse during drying due to capillary forces, similarly to previous observations on emulsion-templated polydimethylsiloxane materials with isolated pores ${ }^{19},{ }^{20}$. Secondly, the capillary forces may deform the matrix and induce the irreversible adhesion between pore walls which decreases the porosity ${ }^{21}$. This mechanism is favored for acrylates which are well known as adhesives ${ }^{22}$. Alternative drying strategies may be used to prevent the collapse, such as supercritical or freeze-drying ${ }^{21,23}$.

\subsection{Sound speed in porous samples with different composition}

Table 3 presents the sound speed in emulsion-templated samples with different matrix composition. In all samples, the sound speed is well below its value in the non-porous matrix (compare to Table 2). This is due to the presence of air-filled porous structure, which decreases drastically the bulk modulus of the material. The sound speed increases when increasing the EHMA/EHA ratio. Rigorous comparison between the samples is difficult because of different porosity. However, as previously observed for porous samples with both hard or soft matrix, the sound speed $C_{\mathrm{L}}$ decreases when $\phi$ increases from 0 to $50 \%$. Thus, in the samples with a partial collapse of pores (X0 and X04), the sound speed is higher than it would be for the $30 \%$ porosity. At similar $\phi$, the sound speed is mainly governed by the $K_{0} / G_{0}$ ratio of the matrix. When increasing the EHMA/EHA ratio, calculation of the $K_{0} / G_{0}$ ratio at the acoustic frequency $f=300 \mathrm{kHz}$ can be determined using the $G_{0}$ values at $300 \mathrm{kHz}$ deduced from Figure 2 and the $K_{0}$ values of the pure matrix deduced from the samples density and acoustic speed $C_{0}$ (Table 1). The obtained $K_{0} / G_{0}$ ratio decreases from 2300 to 27 as the proportion of EHMA increases, revealing the change from soft to hard matrix (see Table 3). Introducing these values in equation (1), one gets the values of the acoustic velocities for porous samples indicated in the last line of Table 3. Comparison between the experimentally measured values and these ones are shown in Figure 5.

Table 3 : Room-temperature acoustic and mechanical properties of porous samples with different matrix composition (the values in italic are calculated as described in the text).

\begin{tabular}{c|c|c|c|c} 
& \multicolumn{4}{|c}{ Samples } \\
\cline { 2 - 5 } & $\begin{array}{c}\text { Porous X0 } \\
\text { with } \Phi=22 \%\end{array}$ & $\begin{array}{c}\text { Porous X04 } \\
\text { with } \Phi=25 \%\end{array}$ & $\begin{array}{c}\text { Porous X07 } \\
\text { with } \Phi=30 \%\end{array}$ & $\begin{array}{c}\text { Porous X1 } \\
\text { with } \Phi=30 \%\end{array}$ \\
\hline $\begin{array}{c}C_{\mathrm{L}}, \mathrm{m} / \mathrm{s} \\
G_{0}, \mathrm{MPa} \\
(300 \mathrm{kHz})\end{array}$ & 175 & 230 & 400 & 440 \\
$K_{0} / G_{0}$ & 1 & 6.3 & 38 & 120 \\
$\begin{array}{c}(300 \mathrm{kHz}) \\
\text { Theoretical } C_{\mathrm{L}} \\
\text { (using eq. }) \mathrm{m} / \mathrm{s}\end{array}$ & 2300 & 368 & 63 & 27 \\
& 78 & 183 & 400 & 695
\end{tabular}


While the overall behavior is similar, an important difference is observed between measurements and theory, especially in the extreme cases of pure EHA and EHMA. We measured the master curves of the porous X0 and X1 samples and compared them with the one obtained for corresponding non-porous samples. As expected for porous polymers ${ }^{3}$, the obtained values of $G^{\prime}$ are smaller compared to those for non-porous ones. For each frequency, the values of $G^{\prime}(\Phi)$ are 2.5 times smaller for porous X0 $(\Phi=22 \%)$ and 3 times smaller for X1 ( $\Phi=30 \%$ ) (Figure 5). These factors can be compared to the one expected by Gibson and Ashby $^{3}$ :

$$
\frac{G^{\prime}(\Phi)}{G_{0}^{\prime}} \approx(1-\Phi)^{2}
$$

From eq. (5), one would expect a decrease by a factor of $\sim 1.7$ for $\mathrm{X} 0$ and 2 for $\mathrm{X} 1$. This indicates that the polymer walls are somehow softer than expected. However, eq. (5) is a simple scaling law and the obtained coefficients still falls in the right range. Once this factor is integrated, the curves of porous and non-porous samples are very similar, and the small differences could not be used to explain the discrepancy of the speed values. Thus, we suggest that the presence of the water during the polymerization leads to a material with the same overall matrix mechanical properties, but distributed non-homogeneously near the pore surface. As discussed in section 3.2, this may be due to the influence of water and surfactant on the polymerization. Thus, we can only conclude that, unlike in the case of PDMS porous samples $^{7,8}$, this simple acoustical model cannot fully explain our results.

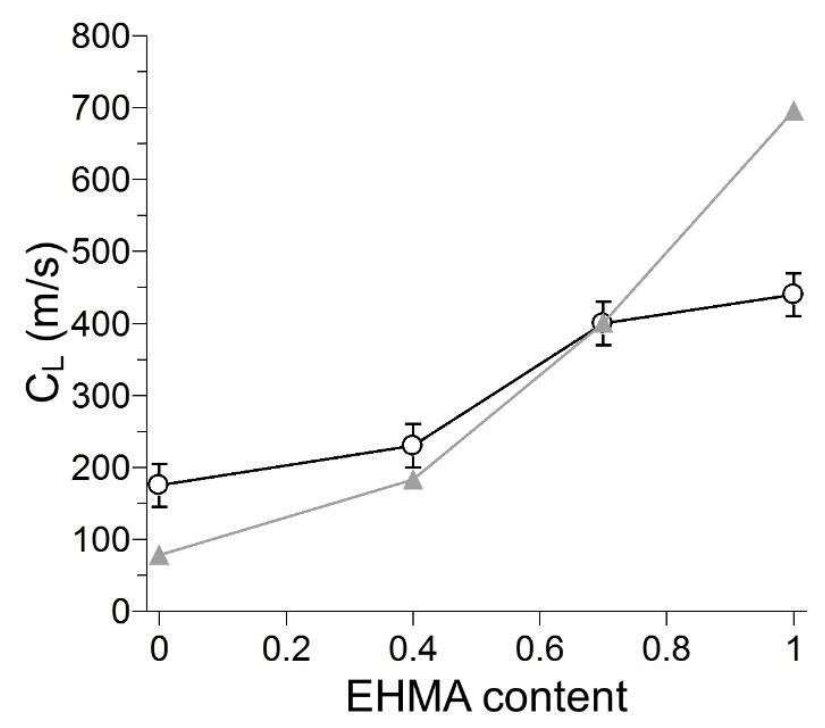

Figure 5-Longitudinal sound velocity in emulsion-templated porous EHA-EHMA samples as a function of EHMA content. Comparison between measured values (open circles) and predicted values from eq. (1) (filled triangles). 


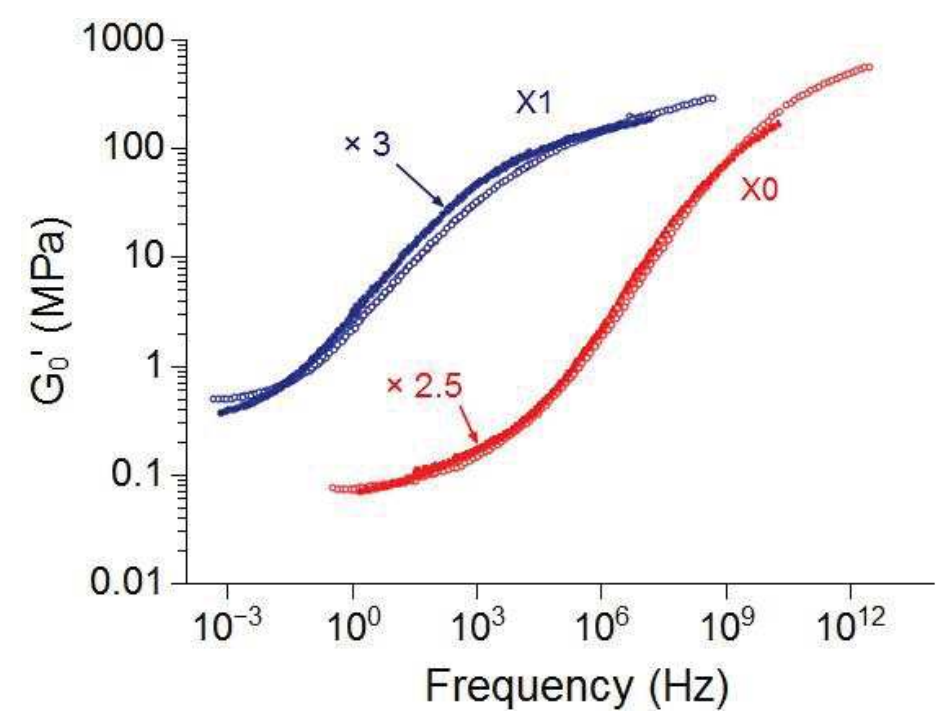

Figure 6 - Comparison of master curves of porous (open symbols) and non-porous (filled symbols) samples for XO (red) and XI (blue). The values of $G_{0}$ ' of porous samples were multiplied by coefficients 2,5 and 3 for $X 0$ and $X 1$, respectively.

\subsection{Effect of temperature on the sound speed in the porous sample X07}

We now comment the results obtained for X07 as temperature is varied from 20 to $62^{\circ} \mathrm{C}$. The values of the sound speed at higher temperatures are given in Table 4 and plotted in Figure 7. Here also, we tried to compare our results with the acoustic model.

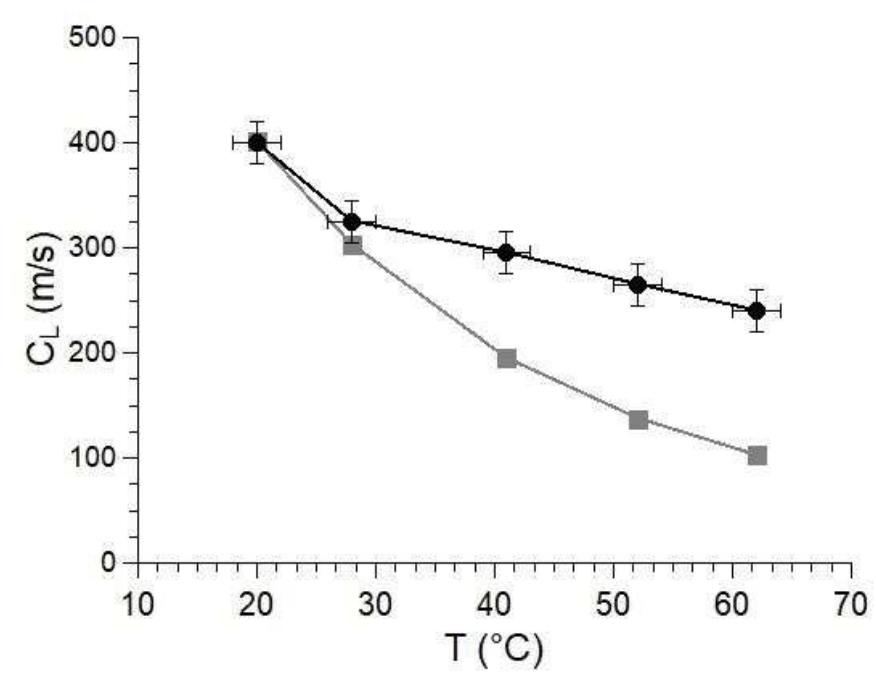

Figure 7 - Longitudinal sound speed in emulsion-templated porous sample X07 as a function of temperature. Comparison between measured values (black circles) and predicted values using equation (1) (gray squares).

To do so, we deduced the values of $G_{0}{ }^{\prime}$ as a function of $T$ using the temperature $(T)-$ frequency $(f)$ equivalence principle:

$$
G_{0}^{\prime}\left(T_{2}, f\right)=G_{0}^{\prime}\left(T_{1}, \frac{a\left(T_{2}\right)}{a\left(T_{1}\right)} \cdot f\right)
$$


where $T_{2}$ and $T_{1}$ are the new and old reference temperatures respectively. We used the Vogel's equation $^{18,24}$ to fit the dependence of shift factors $a(T)$ with temperature:

$$
\log \frac{a\left(T_{2}\right)}{a\left(T_{1}\right)}=\frac{c_{1}^{0}\left(T_{2}-T_{1}\right)}{\left(T_{2}-T_{\infty}\right)}
$$

where $c_{1}{ }^{0}$ and $T_{\infty}$ are the first Williams-Landel-Ferry (WLF) coefficient and Vogel's temperature, respectively. For X07 sample one gets $c_{1}{ }^{0}=11.73$ and $T_{\infty}=180 \mathrm{~K}$ (Figure 8a). The values of $a\left(T_{2}\right)$ were used to plot the master curves at higher temperatures, corresponding to acoustical measurements (Figure $8 \mathrm{~b}$ ). The obtained curves are shifted to higher frequencies with respect to the room-temperature curve, which results in lower values of the $G_{0}$ ' modulus at the same acoustic frequency of $300 \mathrm{kHz}$. The obtained values are given in Table 4.

Table 4 : Acoustic and mechanical properties of the sample X07 at different temperatures (the values in italic are calculated as described in the text).

\begin{tabular}{c|c|c|c|c|c} 
& \multicolumn{5}{|c}{ Porous X07 } \\
& \multicolumn{5}{|c}{ with $\Phi=30 \%$} \\
\hline $\mathrm{T},{ }^{\circ} \mathrm{C}$ & 20 & 28 & 41 & 52 & 62 \\
\hline$C_{\mathrm{L}}, \mathrm{m} / \mathrm{s}$ & 400 & 325 & 295 & 265 & 240 \\
$\begin{array}{c}G_{0}, \mathrm{MPa} \\
(300 \mathrm{kHz})\end{array}$ & 38 & 21 & 8.5 & 4.2 & 2.3 \\
$\begin{array}{c}K_{0} / G_{0} \\
(300 \mathrm{kHz})\end{array}$ & 63 & $113 *$ & $280^{*}$ & $567 *$ & $1034^{*}$ \\
$\begin{array}{c}\text { Theoretical } C_{\mathrm{L}}, \\
\text { (using eq. 1) } \mathrm{m} / \mathrm{s}\end{array}$ & 400 & $303 *$ & $195 *$ & $138^{*}$ & $102 *$
\end{tabular}

* - the value of $K_{0}$ is supposed unchanged with temperature.

Next, we tested equation (1) to predict the sound speed in sample X07 at different temperatures. Concerning $K_{0}$, it is reasonable to consider that its evolution with temperature, even through the glass transition domain, remains very moderate ${ }^{11}$ as compared to the one of $G_{0}^{\prime}$. Thus, we deduce that heating the sample from 20 to $62^{\circ} \mathrm{C}$ should increase the $K_{0} / G_{0}$ ratio by one order of magnitude. However, the theoretical $C_{L}$ curve (gray line in Figure 8 ) predicted using eq. (1) leads to much lower values of the sound speed than the experimental ones. In order to check if our results are reliable, we calculate the experimental sound speed decrease rate $-\mathrm{d} C_{L} / \mathrm{d} T$ which is about $2 \mathrm{~m} / \mathrm{s} \cdot \mathrm{K}$. When divided by the initial sound speed, we obtain the relative sound speed decrease rate $-\left(1 / C_{L}{ }^{20^{\circ} \mathrm{C}}\right) \cdot \mathrm{d} C_{L} / \mathrm{d} T$ of about $0.007 \mathrm{~K}^{-1}$. This value is of the same order as the one reported in non-porous polymers ${ }^{25}$. This means that, the effect of porosity is much lower than expected, an observation for which we currently do not have any explanation. 

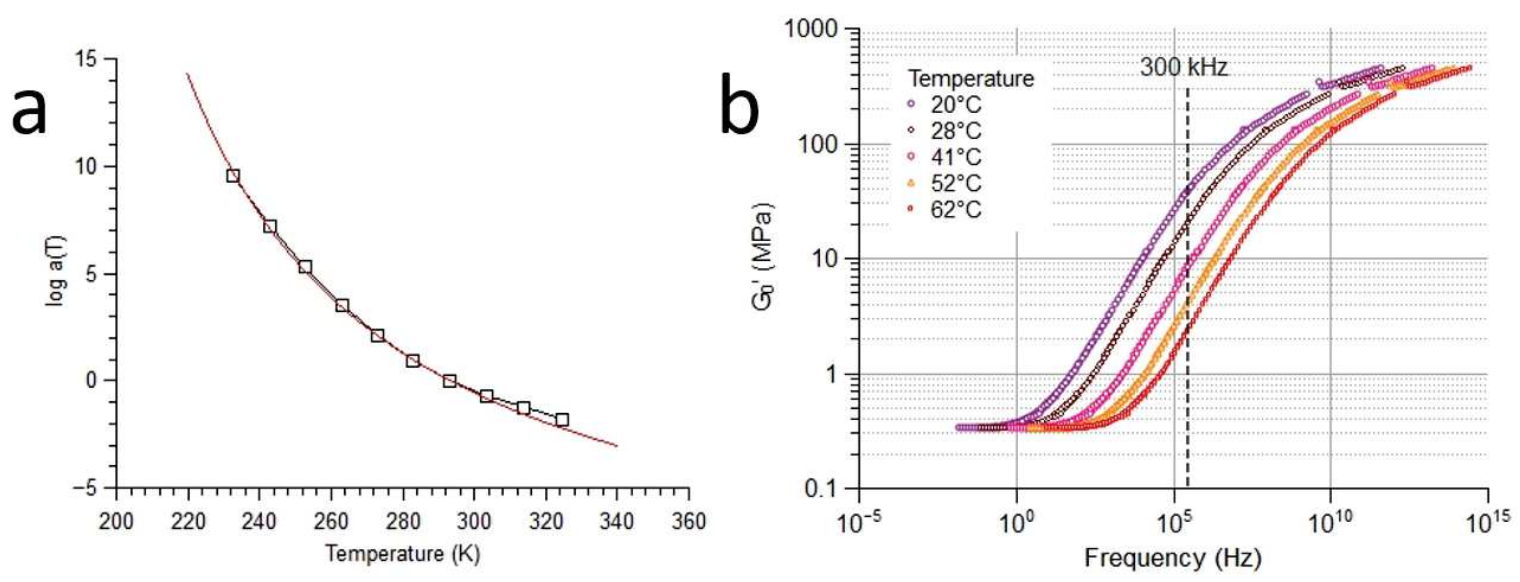

Figure 8 - Shift factors (a) and master curves (b) obtained for the non-porous matrix X07 from the temperature-frequency equivalence principle. The vertical line on the master curve represents the acoustic frequency.

Interestingly, the measurement of the acoustic transmission through the sample as temperature increases led to a dramatic decrease of the signal amplitude (Figure 9) that decreases by a factor of 400 as $T$ varies from 28 to $62^{\circ} \mathrm{C}$. As the system becomes softer, the acoustic transmission through the sample is dramatically affected, which illustrates the tunable aspect of our material. According to the literature ${ }^{2,10}$, this is due to the increase of the loss moduli $K_{0}{ }^{\prime \prime}$ and $G_{0}^{\prime \prime}$.

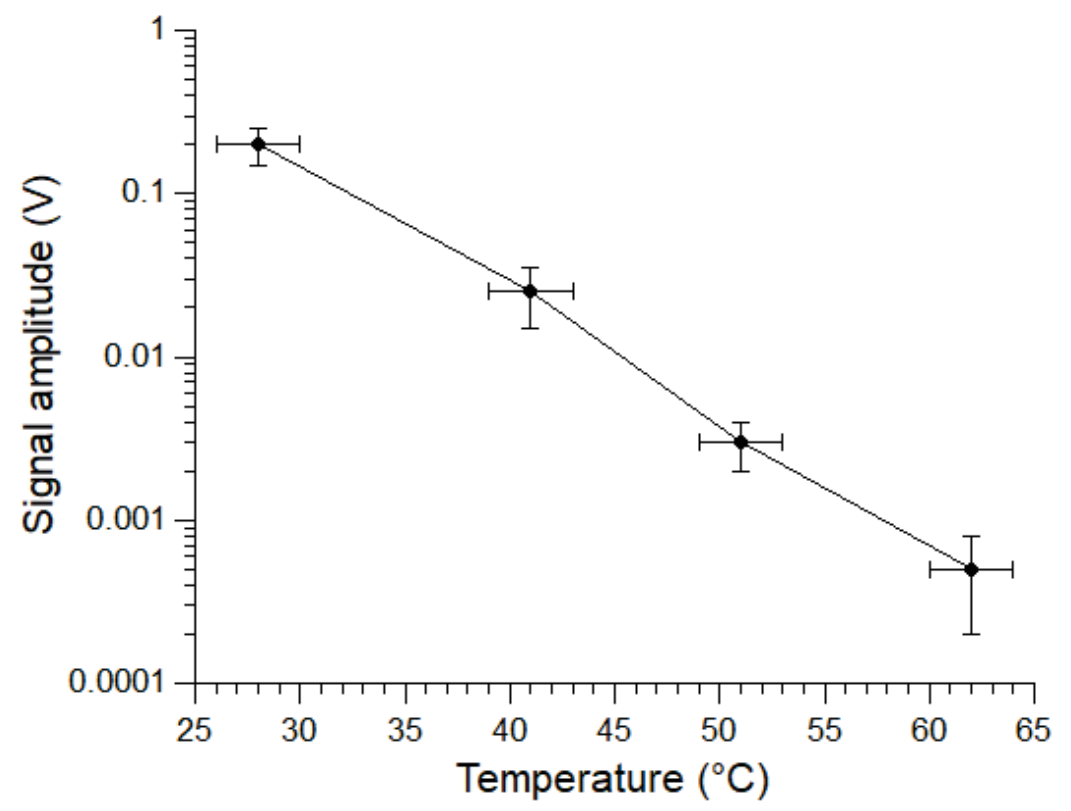

Figure 9 -Evolution of the transmitted amplitude of the acoustical signal in the porous sample X07 as a function of temperature. 


\section{Conclusion}

This study deals with polymer materials with variable glass transition temperatures, obtained by bulk copolymerization of 2-ethylhexyl acrylate (EHA), 2-ethylhexyl methacrylate (EHMA) and the cross-linker ethylene glycol dimethacrylate (EGDMA). Using an emulsiontemplated technique (polyHIPE), we elaborate macroporous materials with variable matrix mechanical and acoustic properties. Our results show that the acoustic properties of these porous materials can be tuned by the composition of the matrix and the temperature. This may be of potential interest in composite ${ }^{26}$ or gradient $^{27}$ acoustic devices.

We first investigate non-porous matrices and show that the increase of EHMA/EHA ratio leads to higher $T_{\mathrm{G}}$ values and, as a consequence, higher shear modulus $G_{0}$ ' at mechanical $(\sim 1$ $\mathrm{Hz})$ and acoustical frequencies $(\sim 300 \mathrm{kHz})$. By applying the temperature-frequency equivalence principle, we build the master curves and deduce the evolution of $G^{\prime}{ }_{0}$ as a function of composition, measurement frequency and temperature. Combined with sound speed measurements in non-porous samples, these data demonstrate the increase of the $K_{0} / G_{0}$ ratio with increasing $T_{\mathrm{G}}$. Using a simple acoustical model, we are able to qualitatively explain the significant increase of the sound speed in porous elastomer materials with increasing $T_{\mathrm{G}}$. We also show that increasing the temperature of a given porous polymer sample leads to a significant decrease of the sound velocity. Beside this, a very strong decrease of the transmitted sound amplitude is observed as the temperature is increased above the glass transition.

Unlike the case of previously published porous PDMS samples, these findings could not be explained quantitatively (using the determined $K_{0}$ and $G_{0}$ values) by a simple acoustical model. We attribute this to the presence of water and surfactant that may affect the polymerization of polyHIPEs leading to different polymer structure and mechanical properties with respect to non-porous matrices.

\section{Acknowledgments}

We thank Kevin David for his help during the first elaboration of the samples. Special thanks to Ahmed Bentaleb for his help in making the device of Figure 2. We are grateful to Franck Collas from Mettler Toledo for his help with DMA and to Mohamed Hanafi for his help with DSC. We also thank Costantino Creton and Nadège Pantoustier for useful discussions. This work was partially funded and performed within the framework of the Labex AMADEUS ANR-10-LABEX-0042-AMADEUS with the help of the French state Initiative d'Excellence IdEx ANR-10-IDEX-003-02. 


\section{References:}

${ }^{1}$ Hierarchically structured porous materials: from nanoscience to catalysis, separation, optics, energy, and life science, ed. Su, B. L.; Sanchez, C.; Yang, X. Y., Eds.; Wiley-VCH Verlag GmbH \& Co. KGaA, Weinheim, Germany. doi: 10.1002/9783527639588.ch10, 2012.

${ }^{2}$ Sound and Vibration Damping with Polymers. 1990. ACS symposium series, Vol. 424, Ed. Corsaro, R. D. ; Sperling, L. H. ISSN 0097-6156.

${ }^{3}$ Gibson, L. J.; Ashby, M. F. Cellular Solids: Structure and Properties, 2nd ed.; Cambridge University Press: Cambridge, 1997.

${ }^{4}$ Combined and Hybrid Adsorbents, Fundamentals and Applications, NATO Security through Science Series C: Environmental Security, ed. J. M. Loureiro and M. T. Kartel, Springer, Dordrecht, 2006.

5 Allard J-F. Propagation of sound in porous media. Modeling sound absorbing materials. Amsterdam: Elsevier; 1993.

${ }^{6}$ Zimmerman, S. Submarine Technology for the $21^{\text {st }}$ Century, Trafford Publishing, 2000.

${ }^{7}$ Brunet, T.; Merlin, A.; Mascaro, B.; Zimny, K.; Leng, J.; Poncelet, O.; Aristégui, C.; MondainMonval, O. Soft 3D acoustic metamaterials with negative index, Nat. Mater., 2015, 14, 384-388.

${ }^{8}$ Ba, A.; Kovalenko, A.; Aristegui, C.; Mondain-Monval, O.; Brunet, T. Soft porous silicone rubbers with ultra-low sound speeds in acoustic metamaterials. Sci. Rep. 2017, 7, 40106.

${ }^{9}$ Kovalenko, A.; Fauquignon, M.; Brunet, T.; Mondain-Monval, O. Tuning the sound speed in macroporous polymers with a hard or soft matrix. Soft Matter, 2017, 13, 4526.

${ }^{10}$ Acoustic Properties. Jarzynski, J.; Balizer, E.; Fedderly, J. J.; Lee, G. in Encyclopedia of Polymer Science and Technology, John Wiley \& Sons, 2003.

${ }^{11}$ Lagakos, N.; Jarzynski, J.; Cole, J. H.; Bucaro, J. A. Frequency and temperature dependence of elastic moduli of polymers. J. Appl. Phys. 1986, 59, 4017.

${ }_{12}$ Alig, I.; Tadjbakhsch, S.; Zosel, A. Comparison of Ultrasonic Shear Wave and DynamicMechanical Measurements in Acrylic-Type Copolymers. J. Polymer Science: Part B, 1998, 36, 17031711.

${ }^{13}$ Lionetto, F.; Maffezzoli, A. Polymer Characterization by Ultrasonic Wave Propagation. Adv. Polymer Technology, 2008, 27, 63-73.

${ }^{14}$ Etchessahar, M.; Sahraoui, S.; Benyahia, L.; Tassin, J. F. Frequency dependence of elastic properties of acoustic foams. J. Acoust. Soc. Am. 2005, 117, 1114.

${ }^{15}$ Guo, X.; Yan, G.; Benyahia, L.; Sahraoui, S. Fitting stress relaxation experiments with fractional Zener model to predict high frequency moduli of polymeric acoustic foams. Mech. Time-Depend. Mater. 2016, 20, 523-533.

${ }^{16}$ Penzel, E.; Rieger, J.; Schneider H.A. The glass transition temperature of random copolymers: 1. Experimental data and the Gordon-Taylor equation. Polymer, 1997, 38, 325-337.

${ }^{17}$ Brandrup, J.; Immergut E. H.; Grulke, E. A. Polymer Handbook, Ed. By John Wiley \& Sons, Inc., Vol. 1, VI, 1999.

${ }^{18}$ Ferry, J. D. Viscoelastic Properties of Polymers; Wiley: New York, 1980.

${ }^{19}$ Kovalenko, A.; Zimny, K.; Mascaro, B.; Brunet, T.; Mondain-Monval, O. Tailoring of the porous structure of soft emulsion-templated polymer materials, Soft Matter, 2016, 12, 5154-5163.

${ }^{20}$ Della Martina, A.; Hilborn, J. G.; Kiefer, J.; Hedrick, J. L.; Srinivasan, S.; Miller, R. D. Siloxane Elastomer Foams. ACS Symp. Ser. 1997, 669, 8-25.

${ }^{21}$ Muralidharan, V.; Hui, C.-Y. Stability of Nanoporous Materials. Macromol. Rapid Commun. 2004, $25,1487-1490$.

${ }^{22}$ Lindner, A.; Lestriez, B.; Mariot, S.; Creton, C.; Maevis, T.; Lühmann, B.; Brummer, R. Adhesive and Rheological Properties of Lightly Crosslinked Model Acrylic Networks. J. Adhesion, 2006, 82, 267-310.

${ }^{23}$ Bisson, A; Rigacci, A; Lecomte, D, Rodier, E, Achard, P; Drying of silica gels to obtain aerogels: Phenomenology and basic techniques, Drying Technologies, 2003, 21, 593-628.

${ }^{24}$ Ahn, D.; Shull, K. R. Effects of Methylation and Neutralization of Carboxylated Poly(n-butyl acrylate) on the Interfacial and Bulk Contributions to Adhesion. Langmuir 1998, 14, 3637-3645. 
${ }^{25}$ Hartmann, B.; Jarzynski, J. Immersion apparatus for ultrasonic measurements in polymers $J$. Acoust. Soc. Am, 1974, 56, 1469.

${ }^{26}$ B. Manzanares-Martínez; J. Sánchez-Dehesa; F. Ramos-Mendieta. Temperature Tunable Acoustic Filter. Twelfth International Congress on Sound and Vibration.

${ }^{27}$ Climente, A.; Torrent, D.; Sanchez-Dehesa, J. Sound focusing by gradient index sonic lenses. Appl. Phys. Lett. 2010, 97, 104103. 
Click here to download Supplementary Material (online publication): SI_Manuscript_Polymer_Kovalenko.pdf 


\section{Highlights}

The mechanical properties of 2-etylhexyl acrylate and 2-ethylhexyl methacrylate polymer matrices are investigated close to the glass transition temperature

Porous polymer materials from 2-etylhexyl acrylate and 2-ethylhexyl methacrylate mixtures are obtained by emulsion templating

Acoustic properties of acrylate porous polymer strongly vary in the glass transition temperature domain

Acoustic transmission through acrylate porous materials strongly decreases as temperature exceeds their glass transition temperature 